\title{
Estudio correlacional entre discapacidad cervical, kinesiofobia y consumo farmacológico en pacientes mayores de 65 años con cervicalgia crónica
}

\author{
Jaime Corral de Toro, César Hidalgo García, José Miguel Tricás Moreno, María \\ Orosia Lucha López, Jacobo Rodríguez Sanz, Cindy Johana Palacio Del Rio, Loreto \\ Ferrández Laliena, Sofía Monti Ballano \\ ${ }^{1}$ Afiliación: Unidad de Investigación de Fisioterapia (UIF) \\ Instituto de Investigación en Ingeniería de Aragón (I3A) \\ Universidad de Zaragoza, Mariano Esquillor s/n, 50018, Zaragoza, Spain.
} Tel. +34-976762707, e-mail:

\section{Resumen}

Estudio correlacional en el cuál se estudiaron las relaciones entre el cuestionario de discapacidad y dolor cervical con el de kinesiofobia y la medicación suministrada en sujetos mayores de 65 años con cervicalgia crónica. Se observó que sí que existe una relación entre el grado de discapacidad cervical, el dolor percibido, las dosis de medicación administradas y la kinesiofobia en los sujetos de la muestra estudiada.

\section{Introducción}

La cervicalgia es una de las principales afecciones músculo-esqueléticas. Su aparición está relacionada, en parte, con el movimiento cervical y la postura que mantiene el sujeto habitualmente (1). Es característico de estos sujetos presentar un rango de movimiento cervical limitado (2). La cervicalgia crónica (duración de los síntomas superior a tres meses) es de las más frecuentes (3). Los síntomas más comunes son: dolor en la región cervical que se irradia a la zona occipital y de los hombros, y aumento de la tensión muscular tanto de la musculatura superficial como de la musculatura profunda. Otros síntomas frecuentes son: vértigos, mareos, afectaciones del rango de movimiento (ROM) y manifestaciones de estrés (4).

En cuanto a la Kinesiofobia se define como un estado del paciente en el cuál presenta un miedo sobredimensionado e irracional al movimiento físico y a la realización de actividad. También se tiene en cuenta la medicación suministrada al paciente, recogiendo cuáles son los medicamentos más utilizados para el dolor de cuello y la frecuencia con la que son usados.

El objetivo fue analizar las posibles correlaciones existentes entre el grado de discapacidad cervical, el dolor percibido, las dosis de medicación administradas y la kinesiofobia en los sujetos de la muestra estudiada

\section{Métodos}

Para conseguir los objetivos, se diseñó un estudio correlacional. Todos los pacientes que cumplieron los criterios de inclusión (mayores de 65 años, firmar el consentimiento informado y presentar dolor cervical de más de tres meses de evolución) y exclusión (presentar signos neurológicos/tumores, marcapasos, fibrilaciones, patología cardiaca, hipertensión no controlada, historia de traumatismo grave/cirugía reciente cervical, patologías sistémicas e inflamatorias, compromiso colágeno congénito, barreras idiomáticas, pendiente de litigio o reclamación judicial, abuso de drogas o problemas psiquiátricos. Se reclutaron como muestra un total de 68 individuos (45 mujeres y 23 hombres), a través de derivación médica, anuncios, redes sociales y clínicas de fisioterapia.

Se recogieron datos de distintas variables modificadoras del efecto (edad, sexo, tiempo de síntomas, actividad laboral, horas de actividad laboral, posición laboral, actividades con cargas, actividad fisica, tareas domésticas, falta de piezas dentarias, férula de descarga, prótesis dentaria, consumo de tabaco, consumo de alcohol, si puede reproducir sus síntomas, horas durante las que realiza tareas en distancia focal, si tiene problemas visuales, toma de analgésicos o antiinflamatorios, presencia de cefales y la frecuencia de las mismas).

Las variables dependientes recogidas fueron: Discapacidad Cervical, se valoró con el cuestionario de Índice de Discapacidad Cervical (IDC) Kinesofobia, se utilizó la Tampa Scale of Kinesiophobia (TSK-17), y dolor Cervical e intensidad del dolor,medida mediante una Escala Visual Analógica (EVA).

\section{Resultados}

Se observó que existe correlación y estadísticamente significativa entre la puntuación obtenida en el cuestionario de kinesiofobia y el dolor percibido por el paciente mediante la EVA. Existe una correlación 
media y estadísticamente significativa entre la puntuación obtenida en el cuestionario de kinesiofobia y la obtenida en el IDC. Existe una correlación media y estadísticamente significativa entre el dolor percibido de media mediante la EVA y la puntuación obtenida en el IDC. Existe una alta correlación y estadísticamente significativa entre el dolor percibido por el paciente mediante la EVA y las veces a la semana que toma medicación. Existe una correlación media y estadísticamente significativa entre la puntuación obtenida en el IDC y las veces a la semana que toma medicación. Existe una correlación media y estadísticamente significativa entre la puntuación obtenida en el cuestionario de kinesiofobia y las veces a la semana que toma medicación.

\section{Discusión}

El objetivo de este estudio era comprobar si existe relación entre el cuestionario de discapacidad y dolor cervical con el de kinesiofobia y la medicación suministrada en sujetos mayores de 65 años con cervicalgia crónica. Los resultados muestran una alta correlación y estadísticamente significativa entre la puntuación obtenida en el cuestionario de kinesiofobia y el dolor percibido por el paciente mediante la EVA. En un estudio se llegó a la conclusión de que el dolor desencadena el miedo al movimiento, provocando a medio plazo discapacidad funcional y desacondicionamiento físico (5).

Se obtuvo una correlación media y estadísticamente significativa entre la puntuación obtenida en el cuestionario de Kinesiofobia y la puntuación obtenida en el índice de discapacidad cervical. Esto coincide con un estudio que indica que altas puntuaciones en este cuestionario está relacionado con tener mayor discapacidad cervical (6).

En cuanto al dolor y a las puntuaciones obtenidas en el IDC, se obtuvo una correlación media y estadísticamente significativa, como se demuestra en un estudio en el cuál se realizó un tratamiento fisioterapéutico de disminución del dolor en cervicalgias (7).

Existe una correlación media y estadísticamente significativa entre la toma de medicación por parte de los pacientes y sus resultados en cuanto a IDC, Kinesiofobia y dolor. La prescripción de medicamentos para combatir el dolor cervical crónico es una de las estrategias más empleadas Numerosos estudios han demostrado que la disminución del dolor y disfunción del paciente mediante fisioterapia se traducen en disminución en la ingesta de fármacos (8).

\section{Conclusión}

Los resultados de este estudio muestran que existe una alta correlación y estadísticamente significativa entre las variables dolor percibido por el paciente mediante la EVA y las veces a la semana que toma medicación y entre la puntuación obtenida en el cuestionario de kinesiofobia y el dolor percibido por el paciente mediante la EVA. Y que existe una correlación media y estadísticamente significativa entre las variables puntuación obtenida en el cuestionario de kinesiofobia y la puntuación obtenida en el IDC; entre el dolor percibido de media mediante la EVA y la puntuación obtenida en el IDC; entre la puntuación obtenida en el IDC y las veces a la semana que toma medicación y entre la puntuación obtenida en el cuestionario de kinesiofobia y las veces a la semana que toma medicación.

1. Vos $T$, Flaxman AD, Naghavi $M$, Lozano $R$, Michaud C, Ezzati M, et al. Years lived with disability (YLDs) for 1160 sequelae of 289 diseases and injuries 1990-2010: a systematic analysis for the Global Burden of Disease Study 2010. Lancet. 2012 Dec 15;380(9859): 2163-96.

2. Woodhouse A, Vasseljen O. Altered motor control patterns in whiplash and chronic neck pain. BMC Musculoskelet Disord [Internet]. 2008;9(1):90.

3. Padilla-Quero E O-PMC. Efectividad de la información escrita e ilustrada en la adherencia a un programa terapéutico domiciliario en pacientes con cervicalgia crónica: un estudio piloto. Fisioterapia. 2013;35(3):105-11.

4. Kaltenborn F. Manual Mobilization of the Joints. Volume II. The Spine. $5^{\text {a }}$ Ed. Norli, Oslo, Norway: OMT España; 2012.

5. Uluğ N, et al.. Comparison of pain, kinesiophobia and quality of life in patients with low back and neck pain. The Journal of Physical Teraphy Science. 2016; 28(2):665-670.

6. Saavedra-Hernández M, Castro-Sánchez AM, Cuesta-Vargas Al, Cleland JA, Fernández-de-lasPeñas C, Arroyo-Morales M. The contribution of previous episodes of pain, pain intensity, physical impairment, and pain-related fear to disability in patients with chronic mechanical neck pain. Am J Phys Med Rehabil. 2012 Dec;91(12):1070-6.

Antúnez L, Almeida R, et al.. Eficacia ante el dolor y la discapacidad cervical de un programa de fisioterapia individual frente a uno colectivo en la cervicalgia mecanica aguda y subaguda. Elsevier. 2017; 49(7):417-425.

Puerma-Castillo MC, García-Ríos MC, PérezGómez ME, Aguilar-Ferrándiz ME, PeraltaRamírez MI. Effectiveness of kinesio taping in addition to conventional rehabilitation treatment on pain, cervical range of motion and quality of life in patients with neck pain: A randomized controlled trial. J Back Musculoskelet Rehabil. 2018;31(3):453-64. 